\section{Future prospects of systemic host modulatory agents in periodontal therapy}

\author{
S. R. Gokhale ${ }^{* 1}$ and A. M. Padhye ${ }^{2}$
}

IN BRIEF
- Reports that various host modulatory
agents have been developed or proposed
to block pathways responsible for
periodontal tissue break down.
Stresses that, currently, subantimicrobial
dose doxycycline (SDD) is the only host
modulatory agent that has been approved
by US food and drug administration and
other regulatory agencies.

Periodontitis is an inflammatory disease caused by microbial infection that leads to destruction of supporting tissues of the teeth. The periodontal tissue destruction is a result of both microbial activity as well as host response. The best chance for clinical improvement may come from implementing complementary treatment strategies that target different aspects of the periodontal balance. Host response modulation, in combination with conventional treatment, offers to restore the balance between health and disease progression in the direction of a healing response. Various host modulatory therapies (HMT) have been developed or proposed to block pathways responsible for periodontal tissue break down. The newer drugs like bortezomib, infliximb, etanercept, vasoactive intestinal peptide, nitric oxide synthase inhibitors and denosumab are developed as a result of better understanding of pathogenesis of inflammatory tissue destruction and may represent the future of periodontal therapy. This review article focuses on the potential systemic host modulatory agents that target cell signalling pathways, cytokines and enzymes.

\section{INTRODUCTION}

Periodontitis is an inflammatory disease of the supporting tissues of the teeth caused by specific microorganisms or groups of specific microorganisms, resulting in progressive destruction of the periodontal ligament and alveolar bone with pocket formation, recession, or both.

Bacteria are always present in the periodontal milieu. The subgingival plaque consists of pathogenic bacteria that release a variety of microbial substances like microbial peptides and bacterial antigens. These substances diffuse across the epithelium into the connective tissue. The interaction of microorganisms with the host determines the course and extent of the resulting disease. Microorganisms may exert pathogenic effects directly by causing tissue destruction or indirectly by stimulating

Senior Lecturer, Department of Periodontology and Implantology, M. A. Rangoonwala College of Dental Sciences and Research Centre, PUNE-411,001, Maharashtra, India; ${ }^{2}$ Professor and Head of Department, Department of Periodontology and Implantology, MGM Dental College and Hospital, Mumbai, Maharashtra, India

${ }^{*}$ Correspondence to: Dr Sneha R. Gokhale Email:drsnehagaikwad@gmail.com

\section{Refereed Paper}

Accepted 26 February 2013

DOI: 10.1038/sj.bdj.2013.432

${ }^{\circledR}$ British Dental Journal 2013; 214: 467-471 and modulating host responses. The host response is mediated by microbial interaction and inherent characteristics of the host, including genetic factors that vary among individuals. In general, the host response functions in protective capacity by preventing local infection from further progression. ${ }^{1}$

It has been recognised that although these bacteria initiate periodontal inflammation the host response is equally important in mediating connective tissue breakdown. Host-derived enzymes like matrix metalloproteinases (MMPs), cytokines and prostanoids cause most of the tissue destruction. ${ }^{2}$

The concentration of these enzymes becomes pathologically high in the periodontal tissues during inflammation. ${ }^{3}$ These pro-inflammatory mediators are counterbalanced by anti-inflammatory mediators like IL-1RA, tissue inhibitors of matrix metalloproteinases (TIMPs), in a condition of health. In the case of a susceptible host, the balance is disturbed leading to excess production of pro-inflammatory mediators and tissue destruction.

The purpose of host modulation therapy (HMT) is to restore the balance between pro-inflammatory and anti-inflammatory mediators by downregulating the destructive mediators and upregulating the protective mediators. Various host modulatory agents have been developed or proposed to block pathways responsible for periodontal tissue breakdown. Specific aspects of disease pathogenesis that have been investigated for modulation include regulation of immune and inflammatory responses, extensive production of matrix metalloproteinases and arachidonic acid metabolites and regulation of bone metabolism. ${ }^{4}$

However, at present, subantimicrobial dose doxycycline (SDD) is the only host modulatory agent that has been approved by US food and drug administration and other regulatory agencies. It includes a dose of $20 \mathrm{mg}$ doxycycline hyclate administered twice daily for a period of three to nine months along with mechanical debridement. The use of SDD has shown clinical benefits with minimal side effects. ${ }^{5}$

The pathogenesis of periodontitis includes an array of inflammatory mediators and pathways that lead to tissue destruction. Therefore, targeting inflammatory mediators like cytokines, cell receptors and cell signalling pathways is necessary to develop effective host modulation therapy. This review of the inflammatory pathways initiating periodontitis, 
and host modulating drugs currently available has highlighted the potential for using other drugs or derivatives of these drugs in the management of periodontal diseases in the future.

\section{DISRUPTION OF CALL SIGNALING PATHWAYS}

Lipopolysaccharide, a major constituent of the cell wall of gram-negative bacteria, is proposed to be a potent stimulator of the process of inflammation. Bacterial lipoprotein/lipopeptides have pathogenspecific molecular patterns. Lipoproteins induce inflammatory cytokine production via various cell signalling pathways.

The cell signalling pathways include the mitogen-activated protein kinase (MAPK)mediated signals, Phosphatidylinositol-3 protein kinase (PI3) pathway, Janus kinase-signal transducer and activator of transcription (JAK-STAT), toll like receptor mediated pathways and nuclear factor-kappa B (NF- $\kappa$ B) activation. ${ }^{6}$ However, so far there is little information about methods to block different cell signalling pathways.

\section{Therapeutic implications}

\section{MAPK inhibitors}

MAPKs are divided into three families - the extracellular signal-regulated kinases (ERK1/2), c-jun N-terminal kinases (JNKs), and p38. Kirkwood et al. ${ }^{7}$ evaluated the effect of p38 MAPK inhibitors in experimental periodontitis in rats and showed significant reduction in bone loss.

\section{Anti-NF- $\mathrm{kB}$ strategy}

Recently, the anti NF-kB treatment strategy has been investigated with some positive results. Transcription factors $\mathrm{NF}-\kappa \mathrm{B}$ are homo or heterodimers found in the cytoplasm of most human cells. Bacteria, bacterial lipopolysaccharide and pro-inflammatory cytokines that is, IL-1 and TNF activate NF- $\kappa$ B. NF- $\kappa$ B activation is regulated primarily through $\mathrm{I}_{\mathrm{K}} \mathrm{B}$ and $\mathrm{I} \kappa \mathrm{B}$ kinase (IKK). In the absence of stimulation, NF- $\kappa B$ is coupled with inhibitory protein, $\mathrm{I} \kappa \mathrm{B}$, which prevents nuclear translocation of NF- $\kappa$ B. Stimulation by a pro-inflammatory cytokine induces recruitment of co-stimulatory cytokines, such as TRAF, which leads to activation of the enzyme NF- $\kappa B$ inducing kinase (NIK).
Thus there are multiple points to inhibit NF- $\kappa \mathrm{B}$ signalling. ${ }^{6}$

Proteasome inhibitors block NF- $\kappa \mathrm{B}$ activation by preventing the degradation of I $\kappa \mathrm{B}$ and therefore the release of NF- $\kappa \mathrm{B}$. One such proteasome inhibitor is bortezomib (Velcade ${ }^{\circledR}$ ).

Bortezomib (Velcade ${ }^{\circledR}$ ) was tested in multiple myeloma with highly promising results and limited side effects. Multiple myeloma is a disease characterised by increased osteoclastic activity that is not balanced by adequate bone formation leading to lytic lesions.

Boissy et $a l .{ }^{8}$ tested bortezomib on cultured osteoclasts. They measured the levels of two bone resorption markers in patients during the three days following five and seven therapeutic bortezomib administrations, respectively. These levels decreased significantly in one to two days after injection and then increased, showing temporary inhibition of osteoclast activity. Thus a direct inhibition of osteoclasts by bortezomib in conditions relevant to treatment of myeloma was observed in this study. ${ }^{8}$

Hongming et al..$^{9}$ investigated the effect of bortezomib on osteoclasts in patients with multiple myeloma. They found that this drug had an inhibitory effect on osteoclasts maturation and function from peripheral blood mononuclear cells. ${ }^{9}$

Since this drug inhibits bone resorption by acting on osteoclasts, it could be used in prevention of bone loss due to periodontitis. However, at present there is insufficient data to support the use of bortezomib in periodontal therapy.

Inhibition of the protein kinases that activate I $\mathrm{KB}$ may also prevent NF- $\mathrm{BB}$ activation. IKK $\beta$ blockade is a promising anti-inflammatory strategy because $N F-\kappa B$ activation depends largely upon activation of the IKK $\beta$ isoform. The IKK (IкB kinase) inhibitor, BMS-345541, was evaluated in the collagen-induced arthritis model in mice by McIntyre et al. in 2003. ${ }^{10}$ When used in a preventive or therapeutic therapy, BMS (Bristol-Myers Squibb)-345541 improved disease activity scores and decreased both synovial inflammation and joint destruction. ${ }^{10}$ However, IKK $\beta$ knockout mice have been shown to develop liver failure related to hepatocyte apoptosis, which is exacerbated in the presence of tumour necrosis factor- $\alpha$.
Despite the pitfalls of systemic antiNF-kB therapy, local administration may be therapeutically and clinically useful. However, further longitudinal clinical trials are necessary to determine the role of anti-NF-kB therapy in the treatment or prevention of periodontal disease.

\section{JAK/STAT pathway inhibitors}

Many cytokines and growth factors exert their biological functions through JAK-STAT signal transduction pathway. Classically, interferons, interleukins and cytokines activate enzymes called Janus kinases (JAK1, JAK2, JAK3 and Tyk2). Activated JAKs, phosphorylate the cytoplasmic domain of the receptor leading the activation of its substrates, especially the proteins known as STATs. These activated proteins then regulate gene transcription. The JAK/STAT pathway plays a significant role in the pathogenesis of rheumatoid arthritis and periodontal disease. ${ }^{11}$

A recent study has shown activation of STAT 3 and STAT 5 proteins in an experimental periodontitis model in rats. However, there is lack of studies with STAT inhibitors in periodontal therapy. ${ }^{12}$

Inhibition of JAK 1 and 3 has proved to be lethal. ${ }^{13}$ At the moment no Tyk2 inhibitor was developed. Targeting Tyk2 would be a useful strategy for the treatment of Th1 mediated disorders such as arthritis. The JAK3 antagonist CP-690550 has shown potential benefits in the treatment of rheumatoid arthritis. ${ }^{14}$

\section{ANTI-CYTOKINE THERAPY}

Host cytokines are the second group of inflammatory mediators, after arachidonic acid metabolites, which are highly implicated in periodontal disease and intensely investigated as potential chemotherapeutic targets. The inflammatory disease process is characterised by domination of proinflammatory cytokine mediators. Therefore, external neutralisation of inappropriate inflammatory cytokines is a therapeutic strategy that has been attempted in many chronic inflammatory conditions, mostly targeting interleukin-1 (IL-1) and tumour necrosis factor- $\alpha$ (TNF- $\alpha$ ), using either monoclonal antibodies or modified receptor proteins. ${ }^{15}$

Currently, anti-cytokine therapy using anti-IL-1 or anti- TNF- $\alpha$ monoclonal antibodies and soluble tumour necrosis factor 
receptors have been approved for the treatment of rheumatoid arthritis, Crohn's disease, juvenile arthritis and psoriatic arthritis with research continuing on periodontal disease. ${ }^{16}$

Two agents considered to be essential in periodontal destruction are IL-1 and tumour necrosis factor- $\alpha$. Therefore, it has been proposed that short-term blockade of IL- 1 and tumour necrosis factor- $\alpha$ may facilitate periodontal wound healing; however, prolonged blockade may have adverse effects.

Under natural conditions an uncontrolled inflammatory response with rapid tissue destruction due to the activities of IL- $1 \beta$ and tumour necrosis factor- $\alpha$ are reversed by the production of anti-inflammatory cytokines such as IL-4, IL-10 and IL-11. ${ }^{17}$

\section{Therapeutic implications}

Blocking the activity of pro-inflammatory cytokines may be a beneficial therapeutic modality for periodontitis. The commercially available anti-cytokine drugs are infliximab, etanercept and anakinra.

Infliximab (Remicade) is a chimeric IgG monoclonal antibody. The term 'chimeric' refers to the use of both mouse (murine) and human components of the drug. TNF- $\alpha$ can also be neutralised with genetically engineered sTNF- $\alpha$-RII. Pers et al. ${ }^{18}$ evaluated periodontal status of 40 patients with rheumatoid arthritis under the treatment of infliximab. They concluded that patients on anti-TNF- $\alpha$ therapy demonstrated less clinical attachment loss and TNF- $\alpha$ blockade could be beneficial in the treatment of periodontitis. $^{18}$

Etanercept (Enbrel) is a fusion protein. It links human soluble TNF receptor to the Fc component of human IgG1. The effect of etanercept was evaluated in experimental periodontitis in rats. Etanercept significantly reduced the degree of:

1. Periodontitis inflammation and tissue injury (histological score)

2. Infiltration of neutrophils (MPO evaluation)

3. iNOS (the expression of nitrotyrosine and cytokines [eg TNF-a])

4. Apoptosis (Bax and Bcl-2 expression). ${ }^{19}$

Anakinra (Kineret) is an interleukin-1 (IL-1) receptor antagonist. It competitively inhibits the binding of IL- 1 to the interleukin-1 type receptor. Anakinra blocks the biological activity of naturally occurring IL-1, including inflammation and cartilage degradation. ${ }^{17}$

Interleukin 11 has been shown to have anti-inflammatory effects by inhibition of TNF- $\alpha$ and other proinflammatory cytokines. ${ }^{20}$ IL-11 directly minimises tissue injury through the stimulation of a tissue inhibitor of metalloproteinases-1 (TIMP-1). ${ }^{21}$ Martuscelli et al. ${ }^{22}$ carried out a study in 2000 , using recombinant human interleukin-11 (rhIL-11) in the treatment of ligature-induced periodontitis in dogs. They found a significant reduction in the rate of clinical attachment loss and radiographic bone loss after rhIL-11 administration twice a week for a period of eight weeks. ${ }^{22}$

A recent systematic review evaluated the effect of anti rheumatic agents on periodontal paramaters and inflammatory biomarkers in rheumatoid arthritis (RA) patients with periodontitis. Limited evidence is available on the effectiveness of anti-TNF- $\alpha$ agents in reducing periodontal inflammation and inflammatory biomarkers in RA subjects with periodontitis. However, a few studies have reported significantly lower levels of IL- $1 \beta$ and TNF- $\alpha$ in serum and GCF in RA patients with antiTNF therapy. ${ }^{23}$

\section{Potential drawbacks of anti-cytokine therapy}

There may be a base line risk factor for malignancy in inflammatory diseases and there are concerns regarding the incidence of malignancies observed in clinical trials of anti-tumour necrosis factor- $\alpha$ therapy. Increased rates of infection such as latent tuberculosis or opportunistic infections like pneumonia are also reported, but the effect of tumour necrosis factor inhibition on the frequency of infection with more common bacterial pathogens is less clear. ${ }^{24}$

There is functional redundancy among the inflammatory cytokines, that is, cytokines function as a network and are produced by different cell types and share overlapping features. Thus, blocking one cytokine will not assure that a receptor mediated response will not be activated by alternate pathways.

All these side-effects stem from the inhibition of essential endogenous factors that play a role in normal physiology.
Anti-tumour necrosis factor- $\alpha$ dampens the host's ability to adequately deal with infection. Detailed characterisation of the biochemical pathways leading to resolution through neutralisation of tumour necrosis factor- $\alpha$ or other immune system pathways requires proper and more extensive studies.

\section{VASOACTIVE INTESTINAL PEPTIDE}

Vasoactive intestinal peptide (VIP) plays a role in immunoregulation. It has been shown to have beneficial immunosuppressive effects in inflammatory and autoimmune conditions. ${ }^{25}$ Linden et al. ${ }^{26}$ studied the changes in the levels of VIP in GCF before and after periodontal treatment. They demonstrated significant reduction in the levels of VIP after periodontal therapy. ${ }^{26}$

Foster et al. ${ }^{27}$ demonstrated that this peptide significantly reduced TNF- $\alpha$ production in human monocytes stimulated with porphyromonas gingivalis lipopolysaccharide. This peptide also inhibited nuclear translocation of nuclear factor-kB and c-Jun. ${ }^{27}$ Foster et al. ${ }^{28}$ also found that VIP inhibits lipopolysaccharide-induced differentiation of monocytes with a concomitant reduction in the expression of toll-like receptor-2 and $-4 .^{28}$

Gürkan et al. ${ }^{29}$ evaluated the therapeutic efficacy of VIP in Escherichia coli lipopolysaccharide-induced experimental periodontitis in rats. They concluded that VIP downregulated the inflammatory response and inhibited alveolar bone loss, possibly by differentially regulating the tissue levels of RANKL and OPG. ${ }^{29}$

There is limited literature on use of VIP in periodontal therapy. The role of VIP as immune-modulator in prevention or treatment of periodontal disease needs to be evaluated by randomised controlled clinical trials and longitudinal studies.

\section{NITRIC OXIDE SYNTHASE INHIBITORS}

Nitric oxide (NO) is a short lived molecule implicated in a wide range of biological processes. NO is produced at high and prolonged concentrations in response to pro-inflammatory stimuli such as lipopolysaccharide via inducible NO synthases (iNOS). High concentrations of NO and peroxynitrite (a product of NO plus superoxide) are cytotoxic to bacteria, fungi, 
protozoa and tumour cells. However, these reactive species may also cause deleterious host effects such as DNA damage, lipid peroxidation, protein damage and stimulation of inflammatory cytokine release. ${ }^{6}$

Excessive nitric oxide and peroxynitrite formation is implicated in the pathophysiology of variety of inflammatory conditions including arthritis, ulcerative colitis, ileitis and circulatory shock.

Lohinai et al. $^{30,31}$ found a reduction of alveolar bone loss and gingival inflammation after the use of a selective iNOS inhibitor mercaptoethylguanidine confirming that NO has a deleterious role in the pathophysiology of periodontitis and that its modulation may prevent tissue destruction. Mercaptoalkylguanidine acts by blocking inducible NO synthases, scavenging peroxynitrite and inhibiting cyclooxygenase enzyme. Lietao et al. ${ }^{32}$ reported that nitric oxide synthase (NOS) inhibitors have protective effects against bone resorption and inflammatory process in ligature-induced periodontitis in rats.

The effect of intragingival injections of S-nitroglutathione was evaluated in experimental periodontitis in rats. S-nitrosoglutathione (GSNO) is a nitric oxide donor that may exert antioxidant, anti-inflammatory and microbicidal actions. It resulted in significant inhibition of alveolar bone loss, increase of bone alkaline phosphatase, decrease of myeloperoxidase activity, as well as significant reduction of inflammatory and oxidative stress markers. ${ }^{33}$

Thus the NOS inhibitors may prove to be potential host modulatory agents that can be used to reduce tissue destruction in periodontitis. However, extensive studies are required to evaluate the side effects and the therapeutic benefit of NOS inhibitors in humans.

\section{RANK/RANKL INHIBITORS}

Normal bone remodeling is controlled by osteoblasts and osteoclasts in a balanced sequence. Receptor activator of nuclear factor $\mathrm{\kappa B}$ (RANK) ligand (RANKL), a member of the tumour necrosis factor family, is expressed on the surface of osteoblasts. RANKL binds the receptor RANK on osteoclast precursors that leads to activation of nuclear factor $\kappa \mathrm{B}$ in the nucleus, inducing differentiation into mature osteoclasts, which degrade or resorb bone. Other osteoclast-activating factors include parathyroid hormone-related protein, interleukins, and chemokines. A decoy receptor for RANKL, osteoprotegerin (OPG), is present in bone marrow and secreted by osteoblasts and acts as a balance between the osteoblasts and osteoclasts. ${ }^{34}$

Denosumab is a humanised IgG2 monoclonal antibody that binds to transmembrane RANKL, preventing RANKL from activating its receptor RANK on the surface of osteoclasts precursors. Neutralising RANKL inhibits osteoclast formation, function and survival and suppresses bone resorption. Denosumab is indicated for the prevention of skeletal-related events in patients with bone metastases from solid tumours. ${ }^{34,35}$

Recently, a randomised controlled clinical trial was conducted to evaluate the effect of denosumab in 36 postmenopausal women with osteoporosis. Periodontal treatment was carried out in the test and control group. At baseline and after treatment, clinical evaluation, hormone blood levels, distance from the crestal alveolar bone (CAB) to the cemento-enamel junction (CEJ), calcaneus bone mineral density (BMD), hormone levels, serum N-telopeptide (NTx), and bone-specific alkaline phosphatase (BSAP) were assessed. Denosumab (DNS) treatment improved periodontal disease and reduced bone turnover markers in postmenopausal women. ${ }^{36}$

The role of RANK/RANKL inhibitors in periodontal therapy is yet to be determined and extensive studies are required to be undertaken. However, these drugs have surely opened newer avenues to periodontal therapy.

\section{CONCLUSION}

Currently, due to an improved understanding of the pathogenesis of periodontal diseases additional approaches to therapy with respect to modulation of the host response has received attention. The concept of modulating host destructive pathways as a strategy for treating periodontal diseases has come a long way since the 1970s. Studies by a number of researchers and clinicians worldwide clearly demonstrate that blocking specific inflammatory mediators and/or enzymes can be efficacious in slowing periodontal disease progression.
The combination of reduction of bacterial load, risk factor modification and host response modification can lead to better clinical outcome as compared to conventional treatment modalities alone.

However, this concept needs to be validated further in controlled clinical trials. As new mediators and pathways of periodontal tissue destruction are identified, so will new host modulating strategies for blocking tissue destruction evolve, which will lead to a bright future for dental healthcare.

1. Page R. The role of inflammatory mediators in the pathogenesis of periodontal disease. J Periodont Res 1991: 26: 230-242.

2. Kantarci A, Hasturk H, Van Dyke T E. Host-mediated resolution of inflammation in periodontal diseases. Periodontol 2000 2006; 40: 144-163.

3. Preshaw P. Host response modulation in periodontics. Periodontol 2000 2008; 48: 92-110.

4. Oringer R J, Research, Science, and Therapy Committee of the American Academy of Periodontology. Modulation of the host response in periodontal therapy. J Periodonto/ 2002; 73: 460-470.

5. Salvi G E, Lang N P. Host response modulation in the management of periodontal diseases. J Clin Peridontol 2005; 32(Suppl 6): 108-129.

6. Kirkwood K L, Cirelli J A, Rogers J E, Giannobile W V. Novel host response therapeutic approaches to treat periodontal diseases. Periodontol 2000 2007; 43: 294-315.

7. Kirkwood K L, Li F, Rogers J E et al. A p38alpha selective mitogen activated protein kinase inhibitor prevents periodontal bone loss. J Pharmacol Exp Ther 2007; 320: 56-63.

8. Boissy P, Andersen T L, Lund T, Kupisiewicz K, Plesner T, Delaissé J M. Pulse treatment with the proteasome inhibitor bortezomib inhibits osteoclast resorptive activity in clinically relevant conditions. LeukRes 2008; 32: 1661-1668.

9. Hongming $H_{\text {, Jian }}$. Bortezomib inhibits maturation and function of osteoclasts from PBMCs of patients with multiple myeloma by downregulating TRAF6. Leuk Res 2009; 33: 115-122.

10. McIntyre K W, Shuster D J, Gillooly K M et al. A highly selective inhibitor of I kappa B kinase, BMS-345541, blocks both joint inflammation and destruction in collagen-induced arthritis in mice. Arthritis Rheum 2003: 48: 2652-2659.

11. Souza J A C, Rossa C Jr, Garlet G P, Nogueira A V , Cirelli J A. Modulation of host cell signalling pathways as a therapeutic approach in periodontal disease. J Appl Oral Sci 2012; 20: 128-138.

12. Garcia de Aquino $S$, Manzolli Leite F R, StachMachado D R, Francisco da Silva J A, Spolidorio L C, Rossa C Jr. Signalling pathways associated with the expression of inflammatory mediators activated during the course of two models of experimental periodontitis. Life Sci 2009; 84: 745-754.

13. Parganas $E$, Wang D, Stravopodis D et al. Jak2 is essential for signalling through a variety of cytokine receptors. Cell 1998; 93: 385-395.

14. West K. CP-690550, a JAK3 inhibitor as an immunosuppressant for the treatment of rheumatoid arthritis, transplant rejection, psoriasis and other immune-mediated disorders. Curr Opin Investig Drugs 2009; 10: 491-504.

15. Elliott M J, Feldmann M, Maini R N. TNF-a blockade in rheumatoid arthritis: rationale, clinical outcomes and mechanisms of action. Int J Immunopharmacol 1995; 17: 141-145.

16. Wooley P H, Dutcher J, Widmer M B, Gillis S. Influence of a recombinant human soluble tumour necrosis factor receptor FC fusion protein on type II collagen induced arthritis in mice. J Immunol 1993; 151: 6602-6607.

17. Waykole Y P, Doiphode S S, Rakhewar PS, Mhaske 
M. Anticytokine therapy for periodontal diseases: where are we now? J Indian Soc Periodontol 2009; 13: 64-68.

18. Pers J O, Saraux A, Pierre R, Youinou P. Anti-TNF-alpha immunotherapy is associated with increased gingival inflammation without clinical attachment loss in subjects with rheumatoid arthritis. J Periodonto/ 2008; 79: 1645-1651.

19. Di Paola R, Mazzon E, Muià C et al. Effects of Etanercept, a tumour necrosis factor-alpha antagonist, in an experimental model of periodontitis in rats. Br J Pharmaco/ 2007; 150: 286-297.

20. Trepicchio W L, Bozza M, Pedneault G, Dorner A J. Recombinant human IL-11 attenuates the inflammatory response through downregulation of proinflammatory cytokine release and nitric oxide production. J Immunol 1996: 157: 3627-3634.

21. Leng S X, Elias J A. Interleukin 11 inhibits macrophage IL-12 production. J Immuno/ 1997; 159: 2161-2168.

22. Martuscelli G, Fiorellini J P, Crohin C C, Howell T H. The effect of IL-11 on the progression of ligature induced periodontal disease in the beagle dog. J Periodontal 2000; 71: 573-578.

23. Han J Y, Reynolds M A. Effect of anti-rheumatic agents on periodontal parameters and biomarkers of inflammation: a systematic review and meta-analysis.
J Periodontal Implant Sci 2012; 42: 3-12.

24. Van der meer J W, Popa C, Netea M G. Side effects of anti-cytokine strategies. Neth J Med 2005; 63: 78-80.

25. Delgado M, Pozo D, Ganea D. The significance of vasoactive intestinal peptide in immunomodulation. Pharmacol Rev 2004; 56: 249-290.

26. Linden G J, Mullally B H, Burden D J et al. Changes in vasoactive intestinal peptide in gingival crevicular fluid in response to periodontal treatment. J Clin Periodontol 2002; 29: 484-489.

27. Foster N, Cheetham J, Taylor J J, Preshaw P M. VIP Inhibits Porphyromonas gingivalis LPS-induced immune responses in human monocytes. J Dent Res 2005; 84: 999-1004.

28. Foster N, Lea S R, Preshaw P M, Taylor J J. Pivotal advance: vasoactive intestinal peptide inhibits upregulation of human monocyte TLR2 and TLR4 by LPS and differentiation of monocytes to macrophages. J Leukoc Biol 2007; 81: 893-903.

29. Gürkan A, Emingil G, Nizam N et al. Therapeutic efficacy of vasoactive intestinal peptide in escherichia coli lipopolysaccharide-induced experimental periodontitis in rats. $J$ Periodontol 2009; 80: 1655-1664.

30. Lohinai Z, Stachlewitz R, Virag L, Szekely A D, Hasko $G$, Szabo $C$. Evidence for reactive nitrogen species formation in the gingivomucosal tissue. J Dent Res 2001; 80: 470-475.

31. Lohinai Z, Mabley J G, Feher E, Marton A, Komjati $\mathrm{K}$, Szabo $\mathrm{C}$. Role of the activation of the nuclear enzyme poly(-ADP-ribose) polymerase in the pathogenesis of periodontitis. J Dent Res 2003; 82: 987-992.

32. Leitao R F, Ribeiro R A, Chaves H V, Rocha FA, Lima V, Brito G A. Nitric oxide synthase inhibition prevents alveolar bone resorption in experimental periodontitis in rats. J Periodonto/ 2005 ; 76: 956-963.

33. Menezes A M, De Souza G F, Gomes A S et al. $S$-Nitrosoglutathione decreases inflammation and bone resorption in experimental periodontitis in rats. J Periodonto/ 2012; 83: 514-521.

34. Kumar A J, Anumala N, Avula H. Novel and often bizarre strategies in the treatment of periodontal disease. J Indian Soc Periodontol 2012; 16: 4-10.

35. Neville-Webbe H L, Coleman R E. Bisphosphonates and RANK ligand inhibitors for the treatment and prevention of metastatic bone disease. Eur J Cancer 2010; 46: 1211-1222.

36. El-Sharkawy H, Elewa M, Elmeadawy S. Does Denosumab affect periodontal bone loss in postmenopausal women? (A randomized placebocontrolled trial). Egypt Dent J 2012; 58: 1321-1325. 